\title{
CHARACTER KERNELS OF DISCRETE GROUPS ${ }^{1}$
}

\section{S. PASSMAN}

Let $G$ be an arbitrary discrete group and let $\Gamma=C[G]$ be its group algebra over the complex numbers $C$. If $\Re$ is an irreducible representation of the algebra then $\Re(\Gamma)=P$ is primitive and hence isomorphic to a dense set of linear transformations over $D$, the commuting ring of $\Re\left[4\right.$, p. 28]. Let $L$ be the center of $D$. If $\operatorname{dim}_{L} P<\infty$ then we say that $\Re$ is finite and since $P$ is central simple over $L[4$, p. 122] we have $\operatorname{dim}_{L} P=m^{2}$. We set $m=\operatorname{deg} \Re$, the degree of $\Re$. If $G$ is finite then $C$ is always the commuting ring of $\Re$ so this agrees with the usual definition of degree.

Again let $P=\Re(\Gamma)$. Then by a theorem of Amitsur [1] deg $\Re \leqq n$ if and only if for every $2 n$ elements $x_{1}, \cdots, x_{2 n}$ in $P$ we have

$$
\left[x_{1}, \cdots, x_{2 n}\right]=\sum^{ \pm} x_{i_{1}} x_{i_{2}} \cdots x_{i_{2 n}}=0 .
$$

The above is known as the standard identity of degree $2 n$. For infinite discrete groups, representation theory is not particularly well behaved. Therefore we will make use of these identities in $C[G]$.

If $g \in G$ we say that $g$ is in the kernel of $\Re$ if and only if $\Re(g)$ $=\Re(1)=1$. We set $\Re_{n}(G)=$ nker $\Re$ where $\Re$ runs over all irreducible representations of degree $>n$. We study groups $G$ with $\Omega_{n}(G)>1$. It is convenient to let $b(G)=$ lub of the degrees of the irreducible representations of $G$. If $b(G) \leqq n$ then trivially $\Omega_{n}(G)=G$. Thus we will be interested mainly in groups with $b(G)>n$.

TheOREM 1. Let $I_{n}=I_{n}[G]$ be the linear subspace of $C[G]$ spanned by all terms of the form $\left[x_{1}, \cdots, x_{2 n}\right]$ with $x_{i} \in C[G]$. Then $g \in \Omega_{n}(G)$ if and only if $(1-g) I_{n}=0$.

Proof. First let $g \in \Omega_{n}(G)$. Let $\Re$ be any irreducible representation of $C[G]$ and consider $\Re\left((1-g) I_{n}\right)$. If deg $\Re>n$ then $\Re(1-g)$ $=0$. If deg $\Re \leqq n$ then $\Re\left(I_{n}\right)=0$. Hence in either case $\Re\left((1-g) I_{n}\right)=0$. Since this holds for all $\Re$ and $C[G]$ is semi simple [5, Theorem 5.2] this yields $(1-g) I_{n}=0$.

Conversely let $(1-g) I_{n}=0$. Let $J_{n}=\left\{a \in C[G] \mid a I_{n}=0\right\}$ so that $J_{n}$ is clearly a left ideal of $C[G]$. To show that it is a right ideal we need only show for $h \in G$ that $J_{n} h \subseteq J_{n}$. Since clearly $h^{-1} I_{n} h=I_{n}$ we have

Received by the editors April 17, 1965.

1 This research was supported, in part, by funds received under NSF Grant GP-3933. 


$$
\left(J_{n} h\right) I_{n}=\left(J_{n} h\right)\left(h^{-1} I_{n} h\right)=J_{n} I_{n} h=0
$$

and so $J_{n}$ is a two-sided ideal. Let $\Re$ be an irreducible representation of $\boldsymbol{C}[G]$ of degree $>n$. Let $V$ be the corresponding left $C[G]$ module and set $V^{*}=\left\{v \in V \mid J_{n} v=0\right\}$. Since $J_{n}$ is a right ideal, $V^{*}$ is a submodule of $V$. Hence either $V^{*}=V$ or $V^{*}=0$. Now deg $\Re>n$ so $\Re(C[G])$ does not satisfy the standard identity of degree $2 n$. Hence $\Re\left(I_{n}\right) \neq 0$ and $I_{n} V \neq 0$. But $J_{n}\left(I_{n} V\right)=0$ so $I_{n} V \subseteq V^{*}$ and hence $V^{*}=V$. Since $(1-g) \in J_{n}$ and $J_{n} V=0$ we have $\Re(g)=\Re(1)$ and the result follows.

Now $I_{n}[G]$ is spanned as a linear space over $C$ by all terms of the form $\left[g_{1}, \cdots, g_{2 n}\right]$ with $g_{i} \in G$. Hence we have the following.

Corollary 2. Let $b(G)>n$. Then $\left|\AA_{n}(G)\right| \leqq \frac{1}{2}(2 n)$ !.

Proof. Now $b(G)>n$ implies that $I_{n}[G] \neq 0$. Thus we can find $g_{i} \in G$ with $\beta=\left[g_{1}, \cdots, g_{2 n}\right] \neq 0$. If $g \in \Omega_{n}(G)$ then $(1-g) \beta=0$ so that $g \beta=\beta$. If $\beta=\sum c_{i} h_{i}$ with $c_{i} \in C$ and $h_{i} \in G$ then $\Omega_{n}(G)$ permutes all those $h_{i}$ with $c_{i}>0$. Thus $\left|\Omega_{n}(G)\right|$ divides $r$, the number of such $h_{i}$. Since $\beta \neq 0$ and $\sum c_{i}=0$ we see that $1 \leqq r \leqq \frac{1}{2}(2 n) !$ and the result follows.

If $H$ is a subgroup of $G$ then $C[H]$ is naturally embedded in $C[G]$. Moreover in this embedding $I_{n}[H] \subseteq I_{n}[G]$. With this remark we have

Corollary 3. Let $H$ be a subgroup of $G$ with $b(H)>n$. Then $\Omega_{n}(G) \subseteq \Omega_{n}(H)$.

Proof. Since $b(H)>n$ there exists $h_{i} \in H$ with $\beta=\left[h_{1}, \cdots, h_{2 n}\right]$ $\neq 0$. Let $g \in \Omega_{n}(G)$. Since $\beta=g \beta$ we see clearly that $g \in\left\langle h_{1}, \cdots, h_{2 n}\right\rangle$ $\subseteq H$. Now $(1-g) I_{n}[G]=0$ implies $(1-g) I_{n}[H]=0$ so $g \in \Omega_{n}(H)$.

The following result essentially reduces the study of groups $G$ with $b(G)>n$ and $\Omega_{n}(G)>1$ to a study of finite groups.

Theorem 4. Let $b(G)>n$ and $\mathfrak{\Omega}_{n}(G)>1$. Let $\beta_{1}, \cdots, \beta_{m}$ be a finite number of nonzero elements of $C[G]$. Then we can find subgroups $H$ and $N$ of $G$ such that

(i) $\beta_{1}, \cdots, \beta_{m} \in C[H]$;

(ii) $b(H)>n$;

(iii) $\Omega_{n}(H)=\Omega_{n}(G)$;

(iv) $N$ is a normal subgroup of $H$ with $\bar{H}=H / N$ finite and $b(\bar{H})>n$;

(v) under the natural homomorphism $\mathrm{C}[H] \rightarrow C[\bar{H}]$ we have $\beta_{i} \rightarrow \bar{\beta}_{i}$ $\neq 0$ and $\Omega_{n}(G) \simeq\left[\Omega_{n}(G)\right]^{-}=\Omega_{n}(\bar{H})$.

Proof. First we show that we can find group elements $g_{i j}$ with 
$i=1,2, \cdots, r$ and $j=1,2, \cdots, 2 n$ such that $g \in \Omega_{n}(G)$ if and only if for all $i$

$$
(1-g)\left[g_{i, 1}, g_{i, 2}, \cdots, g_{i, 2 n}\right]=0 .
$$

Let $\delta$ be the set of all terms $\alpha=\left[g_{1}, \cdots, g_{2 n}\right] \neq 0$ with $g_{i} \in G$. For each such $\alpha \in S$ set $\Re^{\alpha}=\{g \in G \mid(1-g) \alpha=0\}$. As in the proof of Corollary $2,\left|\Omega^{\alpha}\right| \leqq \frac{1}{2}(2 n)$ !. By Theorem $1, \Omega_{n}(G)=\cap \Omega^{\alpha}$. Since each $\Omega^{\alpha}$ is finite, clearly only a finite intersection is required.

For the remainder of the proof fix such a set $\left\{g_{i j}\right\} \subseteq G$. Let $H$ be a finitely generated subgroup of $G$ with $H \supseteq\left\{g_{i j}\right\}$ and $\beta_{1}, \cdots, \beta_{m}$ $\in C[H]$. Such groups clearly exist. Clearly $b(H)>n$ and we have $\Omega_{n}(H) \supseteq \Omega_{n}(G)$. But if $h \in \Omega_{n}(H)$ then $(1-h)\left[g_{i, 1}, \cdots, g_{i, 2 n}\right]=0$ for all $i$ so $h \in \Omega_{n}(G)$. Hence $\Omega_{n}(H)=\Omega_{n}(G)$. With this choice of $H$ we have (i), (ii) and (iii) of Theorem 4 satisfied.

We now show that $H$ is a subdirect product of finite groups. Fix $g \in \Omega_{n}(H)$ with $g \neq 1$. Let $h$ be any nonidentity element of $H$. In $C[H]$ the expression $\gamma=(1-g)(1-h)$ is nonzero since otherwise $1+g h=g+h$ and so $1=g$ or $h$. Since $C[H]$ is semisimple there exists an irreducible representation $\Re$ of $C[H]$ with $\Re(\gamma) \neq 0$. Hence $\Re(g)$ $\neq 1$ and $\Re(h) \neq 1$. The first of these implies that deg $\Re \leqq n$. Hence we conclude that $H$ is a subdirect product of linear groups of finite degree. By Proposition 7.3 of [3], each such linear group being finitely generated is the subdirect product of finite groups. Hence the result follows.

Now only a finite number of group elements of $H$ occur in the expressions for the $\beta_{i}$ and the $\left[g_{i, 1}, \cdots, g_{i, 2 n}\right]$. Let these be $h_{1}, \cdots, h_{s}$. Then we can write $\beta_{i}=\sum c_{i j} h_{j}$ and $\left[g_{i, 1}, \cdots, g_{i, 2 n}\right]=\sum d_{i j} h_{j}$ with $c_{i j}, d_{i j} \in C$. Let $\mathfrak{J}$ be the finite set containing (1) $\Omega_{n}(H),(2)$ all elements of the form $h_{j} h_{k}^{-1}$, and (3) all elements of the form $h_{j} h_{j^{\prime}}^{-1} h_{k^{\prime}} h_{k}^{-1}$. By the above there exists a normal subgroup $N$ of $H$ of finite index with $N \cap J=\{1\}$. We show now that with this $N$, (iv) and (v) of Theorem 4 follow.

Since $N \cap J=\{1\}$ and $h_{j} h_{k}^{-1} \in J$ it follows that under the homomorphism $H \rightarrow \bar{H}=H / N$, that $\bar{h}_{j}$ (the image of $h_{j}$ ) is not equal to $\bar{h}_{k}$. With this we see that $\bar{\beta}_{i} \neq 0$ and that $\left[\bar{g}_{i 1}, \cdots, \bar{g}_{i 2 n}\right] \neq 0$. The latter implies in addition that $b(\bar{H})>n$. Since any irreducible representation of $C[\bar{H}]$ can be viewed as one of $C[H]$ we have $N \Omega_{n}(H) / N$ $\subseteq \Omega_{n}(\bar{H})$. But $N \Omega_{n}(H) / N \simeq \Omega_{n}(H) /\left(N \cap \Omega_{n}(H)\right) \simeq \Omega_{n}(H)$ since $N \cap \Omega_{n}(H)=1$. Hence $\Omega_{n}(H)=\Omega_{n}(G)$ is contained isomorphically in $\Omega_{n}(\bar{H})$. We need only show that the isomorphism is onto. Let $\bar{g} \in \Omega_{n}(\bar{H})$ with $g$ an inverse image of $\bar{g}$. It suffices to show that $g \in N \Omega_{n}(H)$. 
Since $\bar{g} \in \Omega_{n}(\bar{H})$ we have for all $i$

$$
\bar{g}\left[\bar{g}_{i 1}, \cdots, \bar{g}_{i 2 n}\right]=\left[\bar{g}_{i 1}, \cdots, \bar{g}_{i 2 n}\right] .
$$

In $C[G]$ this yields clearly

$$
g\left(\sum d_{i j} h_{j}\right)=\sum d_{i j} n_{i j} h_{j}
$$

with $n_{i j} \in N$. This follows since all the $\bar{h}_{j}$ are distinct. We show now that all the $n_{i j}$ are equal. Consider one such element $n_{i j} h_{j}$. This comes from a term $g h_{j^{\prime}}$ on the left of the above equation. Thus $g h_{j^{\prime}}=n_{i j} h_{j}$ or $g=n_{i j} h_{j} h_{j^{\prime}}^{-1}$. Replacing $i$ by $i^{\prime}, j$ by $k$, and $j^{\prime}$ by $k^{\prime}$ we also have $g=n_{i^{\prime} k} h_{k} h_{\boldsymbol{k}^{\prime}}^{-1}$. Thus

$$
n_{i j}^{-1} n_{i^{\prime} k}=h_{j} h_{j^{\prime}}^{-1} h_{k^{\prime}} h_{k}^{-1} \in N \cap J=\{1\}
$$

so $n_{i j}=n_{i^{\prime} k}$. Let their common value be $n_{11}$. Then for all $i$

$$
n_{11}^{-1} g\left[g_{i 1}, \cdots, g_{i 2 n}\right]=\left[g_{i 1}, \cdots, g_{i 2 n}\right] \text {. }
$$

By the choice of the $g_{i j}$ this implies that $n_{11}^{-1} g \in \Omega_{n}(G)=\Omega_{n}(H)$ and $g \in N \Omega_{n}(H)$. This completes the proof.

As an application of the above result we prove

Theorem 5. Let $\Re_{n}(G)>1$. Then $b(G) \leqq n^{2}$.

Proof. Suppose by way of contradiction that $b(G)>n^{2}=m$. Then we can find group elements $g_{1}, \cdots, g_{2 m}$ such that $\left[g_{1}, \cdots, g_{2 m}\right] \neq 0$. Set $\beta_{i}=g_{i}$ and $\beta_{2 m+1}=\left[g_{1}, \cdots, g_{2 m}\right]$. Applying Theorem 4 we obtain a finite group $\bar{H}$ with $\Omega_{n}(\bar{H})>1$ and containing elements $\bar{g}_{1}, \cdots, \bar{g}_{2 m}$ with $\left[\bar{g}_{1}, \cdots, \bar{g}_{2 m}\right] \neq 0$. Hence $b(\bar{H})>n^{2}$.

Let $\bar{h} \in \Omega_{n}(\bar{H})$ with $\bar{h} \neq 1$. Let $\theta$ be an irreducible complex character of $\bar{H}$ of degree $>n^{2}$. This exists since $b(\bar{H})>n^{2}$. Clearly $\bar{h}$ is in the kernel of $\theta$, that is $\theta(\bar{h})=\theta(1)=\operatorname{deg} \theta$. Since $C[\bar{H}]$ is semisimple we can find an irreducible character $\chi$ of $\bar{H}$ with $\bar{h}$ not in the kernel of $\chi$. Let $\theta \chi=\sum a_{i} \chi_{i}$ where the $\chi_{i}$ are irreducible. Since $\bar{h} \notin$ kernel of $\theta \chi$ there exists a $\chi_{i}$, say $\chi_{1}$ with $\bar{h} \notin$ kernel $\chi_{1}$. Now $\chi_{1}$ is a constituent of $\theta \chi$ so

$$
1 \leqq\left[\theta \chi, \chi_{1}\right]=\left[\theta, \bar{\chi} \chi_{1}\right]
$$

where [ , ] denotes the usual inner product of characters. Hence $\theta$ is a constituent of $\bar{\chi} \chi_{1}$. This yields

$$
n^{2}<\operatorname{deg} \theta \leqq \operatorname{deg} \bar{\chi} \chi_{1}=(\operatorname{deg} \chi)\left(\operatorname{deg} \chi_{1}\right) .
$$

Clearly at least one of $\operatorname{deg} \chi$ or $\operatorname{deg} \chi_{1}$ is $>n$ and this is the required contradiction. 
By Theorem $\mathrm{F}$ of [3] groups $G$ with $b(G) \leqq n^{2}$ all have abelian subgroups of index $\leqq J\left(2 n^{2}\right)$, where $J$ is the function associated with Jordan's theorem on finite linear groups. Thus $\Re_{n}(G)>1$ is a rather restrictive condition for a group to satisfy. We discuss now a method of constructing a class of groups $G$ with $b(G)>n$ and $\Omega_{n}(G)>1$.

Let $p$ be a fixed prime and let $e \geqq p$. Suppose we have $e$ groups $H_{i}$ each having a central subgroup $Z_{i}=\left\langle z_{i}\right\rangle$ of order $p$. Set

$$
\begin{aligned}
a_{i} & =\text { minimal degree of irreducible character } \theta_{i} \text { of } H_{i} \text { with } Z_{i} \nsubseteq \operatorname{ker} \theta_{i} ; \\
b_{i} & =b\left(H_{i}\right) ; \\
c_{i} & =b\left(H_{i} / Z_{i}\right) .
\end{aligned}
$$

We suppose further that for all $i$

$$
\left(b_{i} / c_{i}\right)>\prod_{j=1}^{e}\left(b_{j} / a_{j}\right)
$$

Let $U$ be an abelian group of type $(p, p)$ generated by $u, v \in U$. We define a homomorphism of

$$
Z_{1} \times Z_{2} \times \cdots \times Z_{e} \rightarrow U
$$

by $z_{i} \rightarrow u v^{i}$. This is clearly onto. Let the kernel be $N$. Then $N$ is a central and hence normal subgroup of $H=H_{1} \times H_{2} \times \cdots \times H_{e}$. Set $G=H / N$ so that $G \supseteq U$, a central subgroup of type $(p, p)$. Set $n+1$ $=\prod a_{i}$. We show that $v \in \Omega_{n}(G)$ and that $b(G)>n$.

Let $\theta$ be an irreducible character of $G$. Then since $U$ is central $\theta \mid U=(\operatorname{deg} \theta) \lambda$ where $\lambda$ is a linear character of $U$. Hence some subgroup of order $p$ of $U$ is the kernel of $\theta$. The subgroups of $U$ are of course $\langle v\rangle$ and $\left\langle u v^{i}\right\rangle$ for $i=1,2, \cdots, p$. Since $G$ is a homomorphic image of $H, \theta$ can be viewed as a character of $H$. In $H$ we write $\theta=\theta_{1} \theta_{2} \cdots \theta_{e}$ where $\theta_{i}$ is an irreducible character of $H_{i}$.

Suppose first that $\langle v\rangle \nsubseteq \operatorname{ker} \theta$. Then for some $i=1,2, \cdots, p$ we have $\left\langle u v^{i}\right\rangle \subseteq \operatorname{ker} \theta$. Then clearly in $H, z_{i} \in \operatorname{ker} \theta_{i}$. Hence $\operatorname{deg} \theta_{i} \leqq c_{i}$ and of course for $j \neq i, \operatorname{deg} \theta_{j} \leqq b_{j}$. Thus

$$
\operatorname{deg} \theta \leqq\left(\prod b_{j}\right)\left(c_{i} / b_{i}\right)<\prod a_{j}=n+1 .
$$

Hence $\operatorname{deg} \theta \leqq n$ and $v \in \Omega_{n}(G)$. Now choose $\theta$ to be a character of $G /\langle v\rangle$ which is faithful on cyclic $U /\langle v\rangle$. Viewed in $H$ we see that for all $i, z_{i} \notin \operatorname{ker} \theta_{i}$. Hence $\operatorname{deg} \theta_{i} \geqq a_{i}$ and so $\operatorname{deg} \theta \geqq \prod a_{i}=n+1$. Therefore $b(G)>n$ and the result follows.

Using the above we can easily construct some examples.

EXAMPLE 6. Let each $H_{i}$ be a nonabelian group of order $p^{3}$. Then 
$a_{i}=p, b_{i}=p$ and $c_{i}=1$ and so equation (1) is satisfied. This yields groups $G$ nilpotent of class 2 .

Indecomposable nonnilpotent groups with nontrivial kernels can be obtained as follows.

EXAmple 7. Let $P$ denote the quaternion group of order 8 if $p=2$ or the nonabelian group of order $p^{3}$ and period $p$ if $p$ is odd. Let $Z$ be the center of $P$. We have $|Z|=p$. Let $A$ denote the group of automorphisms of $P$ which centralize $Z$. $A$ is easily seen to be isomorphic to the Symplectic group $S_{p_{2}}(p)$ whose order is $p(p+1)(p-1)$. Let $\alpha \in A$ be of prime order $q \neq p$ and let $H$ be the semidirect product of $P$ by the cyclic group $\langle\alpha\rangle$. Thus $P$ is normal in $H$ with index $q$ and $Z$ is central in $H$.

Let $\chi$ be an irreducible character of $H$. By Proposition 1.2 of [2], either $\chi \mid P$ is irreducible or $\chi \mid P$ is the sum of $q$ conjugate characters under the action of $\langle\alpha\rangle$. In the first case deg $\chi=1$ or $p$. In the second case let $\phi$ be an irreducible constituent of $\chi \mid P$. If $\operatorname{deg} \phi=p$ then $\phi$ vanishes off $Z$. Since $\alpha$ centralizes $Z, \phi^{\alpha}=\phi$, a contradiction. Hence $\operatorname{deg} \phi=1$ and $\operatorname{deg} \chi=q$. Thus $H$ has characters of degree $1, p$ and $q$ only. Moreover if $H=H_{i}$ we have easily $a_{i}=p, b_{i}=\max (p, q)$ and $c_{i}=q$.

If $p=2$ choose $q=3$. Then $b(H)=3$ and $Z \subseteq \Omega_{2}(H)$. If $p>2$ then choose $q$ to divide $p(p+1)(p-1)$ so $p>q$. Hence in this case equation (1) is satisfied and the group $G$ constructed has the required property.

\section{REFERENCES}

1. S. A. Amitsur, Groups with representations of bounded degree. II, Illinois J. Math. 5 (1961), 198-205.

2. I. M. Isaacs and D. S. Passman, Groups with representations of bounded degree, Canad. J. Math. 16 (1964), 299-309.

3. - Characterization of groups in terms of the degrees of their characters, Pacific J. Math. 15 (1965), 877-903.

4. N. Jacobson, Structure of rings, Amer. Math. Soc. Colloq. Publ. Vol. 37, Amer. Math. Soc., Providence, R. I., 1956; rev ed., 1964.

5. C. Rickart, Uniqueness of norm in Banach algebras, Ann. of Math. 51 (1950), 615-628.

University of CALifornia, Los Angeles 